\title{
Wave dissipation by ion cyclotron resonance in the solar corona
}

\author{
C.-Y. Tu ${ }^{1,2}$ and E. Marsch ${ }^{1}$ \\ 1 Max-Planck-Institut für Aeronomie, 37191 Katlenburg-Lindau, Germany \\ ${ }^{2}$ Department of Geophysics and State Key Laboratory for Turbulence Research, Peking University, Beijing, \\ 100871, China
}

Received 2 November 2000 / Accepted 19 December 2000

\begin{abstract}
It has recently been suggested that small-scale reconnection occurring in the chromospheric network creates high-frequency Alfvén waves, and that these waves may represent the main energy source for the heating of the solar corona and generation of the solar wind. However, if these waves exist, they will be absorbed preferentially by the minor heavy ions with low gyrofrequencies, and thus it is unclear whether there is actually enough wave energy left over for the heating and acceleration of the major solar wind ions, namely protons and alpha particles, in the extended corona after the absorption by heavy ions (Cranmer 2000). We have studied this problem with the multi-fluid model presented by Tu \& Marsch (2001), which includes the self-consistent treatment of the damping of the waves as well as the associated acceleration and heating of the ions. We found that if the wave power density is sufficiently large, say about $1000 \mathrm{nT}^{2} \mathrm{~Hz}^{-1}$ at $160 \mathrm{~Hz}$ and $2.5 R_{\odot}$, then the wave absorption by a prominent minor ion such as $\mathrm{O}^{+5}$ is small, and most of the wave energy is left for absorption by protons. This occurs because the minor ions are quickly (within several gyroperiods) accelerated and then are induced to partially surfing the waves. However, if the wave power is too low, say lower than $10 \mathrm{nT}^{2} \mathrm{~Hz}^{-1}$ at $160 \mathrm{~Hz}$ and $2.5 R_{\odot}$, then the damping of the wave power by the $\mathrm{O}^{+5}$ ions is severe, and little wave energy is left for protons.
\end{abstract}

Key words. Sun: corana - Sun: general

\section{Introduction}

High-frequency Alfvén waves created by small-scale reconnection in the Sun's chromospheric network are considered (Axford \& McKenzie 1997) as a possible energy source of the solar corona and the solar wind. Based on this idea, McKenzie et al. $(1995,1997)$ developed and evalulated a model for the fast solar wind. Tu \& Marsch (1997), Marsch \& Tu (1997a,b) and Hackenberg et al. (1999, 2000) further developed two-fluid models for coronal heating and wind acceleration, whereby the energy was supplied by high-frequency Alfvén waves propagating outward from the coronal base in a coronal funnel and coronal hole. The evolution of the wave spectrum was determined selfconsistently. The models were able to produce reasonable parameters for the corona and wind, in compliance with basic observations of the effective wave amplitude as derived from the line width of extreme ultraviolet radiation lines (Wilhelm et al. 1998) that are emitted by heavy ions in the solar transition region. Tu \& Marsch (2001) have further used the high-frequency wave-energysweeping mechanism of the previous models to explain also why the $\mathrm{O}^{+5}$ line widths indicate strong perpendicular ion heating (Kohl et al. 1998).

Send offprint requests to: E. Marsch, e-mail: marsch@linmpi.mpg.de
However, the wind model with energy supply from high-frequency waves is far from being fully established. At the present time there exist no direct measurements that could give us direct information about high-frequency waves at the coronal base. The survival probability of such waves out to about $5 R_{\odot}$ may be evaluated by the depolarization effects they would have on radio waves, as was suggested and discussed by Spangler \& Mancuso (2000). Yet, they found no sizable Faraday screen depolarization of the originally polarized stellar radio waves, when they propagated through the 5 to 8 solar radii distance interval in the near-Sun solar wind. This result is consistent with the model results obtained by Tu \& Marsch (1997) and Marsch \& Tu (1997a), because the tiny amplitudes of Alfvén waves at high frequencies (see, e.g., the values at $5 R_{\odot}$ in their Table 2 ) should not give any observable depolarization effect (see the Fig. 3 in Spangler \& Manusco 2000). In any case, one cannot derive reliable information about the wave dissipation alone from those waves surviving the transit into interplanetary space, without having the full information about the total input of waves at the coronal base.

Besides the question of the existence of high-frequency waves, there is another difficult problem. How is the energy of the high-frequency waves distributed to the different ions, when they are all absorbing wave energy at 
their respective cyclotron resonance? Because the resonant damping rate of the waves is very high, the ions with a low resonance frequency may absorb most of the energy in a short time, and then little is left for protons. However, SOHO observations (Kohl et al. 1998) showed that both $\mathrm{O}^{5+}$ ions and protons are heated, and that heating is indeed needed to explain the widths of EUV emission lines (Wilhem et al. 1998). To avoid the problem mentioned above, Tu \& Marsch (1999) and Tu et al. (1999) suggested a two-step process yielding sizable heating of both $\mathrm{O}^{+5}$ and protons. The essential idea is that while being accelerated, the $\mathrm{O}^{+5}$ ions become resonant with cyclotron waves at ever higher waves numbers, at which a considerable number of the protons are also resonant with the waves and thus compete with the minor ions for wave power. When using higher values of the Alfvén speed than used in that paper, only fewer protons can resonate with waves at the Doppler-shifted frequency of the $\mathrm{O}^{+5}$ ions, as was pointed out by Cranmer (2000).

Gomberoff et al. (1996) showed that the acceleration of the ions as a result of cyclotron resonance makes them partially surf the waves and hinders them from absorbing the waves. The waves will then become resonant with ions at higher resonance frequencies. However, they did not calculate the damping of the waves. Cranmer (2000) argued that the high-frequency waves will damp out rapidly by resonance with a multitude of heavy ions before being able to resonate with the majority species protons and alpha particles in the corona. It is found that high-frequency (10-1000) $\mathrm{Hz}$ waves propagating upward from the base of the corona are damped significantly when they resonate with those ions having a low charge-to-mass ratios of about 0.1 , and thus at greater heights only a negligible amount of waves to resonate with would be available for the ions with higher charge-to-mass ratios.

Here we will concentrate on this problem. In the estimation of Cranmer (2000), constant ion velocities were assumed. We treat the ion acceleration and heating selfconsistently and include wave damping, using the multifluid model developed by Tu \& Marsch (2001), based on earlier work by Marsch et al. (1982). We find that if the power density of the high-frequency waves is very small, wave energy will mostly be absorbed by heavy ions. However, if the wave spectrum is reasonably intense, the $\mathrm{O}^{+5}$ ions quickly start partially surfing the waves and their absorption becomes very small. In Sect. 2, we describe the model, especially the dispersion relation we used in the numerical calculations. In Sect. 3, we present the numerical results for three different levels of the wave amplitude. In Sect. 4, we discuss the main results and our conclusions.

\section{Model description}

In our calculations, we use the two-ion-fluid equations for Model 3 in Tu \& Marsch (2001), including the continuity equations, momentum equations, and the energy equations with anisotropic temperature for $\mathrm{O}^{+5}$ ions and isotropic temperature for protons. The heating rates, the acceleration rates and the wave damping rates are calculated by using the quasilinear theory for cyclotron resonance, as given by Tu \& Marsch (2001). The evolution of the wave spectrum versus radial distance is calculated by using the WKB-law modified by the resonant damping. The time-stationary set of equations is integrated from $2.5 R_{\odot}$ to $3.7 R_{\odot}$. The boundary conditions are as follows: fluid velocity, $u_{\mathrm{O}^{+5}}=340 \mathrm{~km} \mathrm{~s}^{-1}$, thermal velocities, $v_{\|, \mathrm{O}^{+5}}=260 \mathrm{~km} \mathrm{~s}^{-1}, v_{\perp, \mathrm{O}^{+5}}=120 \mathrm{~km} \mathrm{~s}^{-1}$, oxygen relative abundance, $\eta=n_{\mathrm{O}^{+5}} / n_{\mathrm{p}}=10^{-6}$. The slope of the wave spectrum at the boundary was assumed to be -2 . The spectrum density is set to zero below a lower limit, which is at a frequency slightly lower than the cyclotron resonance frequency of $\mathrm{O}^{+5}$. The other parameters, such as the background magnetic field and electron temperature, are the same as in the paper by Tu \& Marsch (2001).

We use the dispersion relation of left-hand-polarized cyclotron waves in a cold plasma containing $\mathrm{O}^{+5}$ ions, protons and electrons. The dispersion equation (after Gomberoff et al. 1996) may be written as

$y^{2}=\frac{x^{\prime 2}}{1-x^{\prime}}+\frac{16 \cdot 5 \eta\left(x^{\prime}-y \Delta u_{\mathrm{O}^{+5}} / V_{\mathrm{A}}\right)^{2}}{5-16\left(x^{\prime}-y \Delta u_{\mathrm{O}^{+5}} / V_{\mathrm{A}}\right)}$,

where $x^{\prime}=\omega^{\prime} / \Omega_{\mathrm{p}}$, and $y=k_{\|} V_{\mathrm{A}} / \Omega_{\mathrm{p}}$. Here $\omega^{\prime}$ is the frequency in the rest frame of the solar wind, whereas $\omega$ is the wave frequency in the inertial (solar) frame of reference, and $\Delta u_{\mathrm{O}^{+5}}=u_{\mathrm{O}^{+5}}-u_{\mathrm{s}}$. Here $u_{\mathrm{s}}$ is the solar wind speed, which is about the proton speed $u_{\mathrm{p}}$, i.e. $u_{\mathrm{s}} \cong u_{\mathrm{p}}$, in the present calculation. Since the abundance $\eta$ is very small, the solution of the dispersion equation may be obtained approximately as follows. We calculate the cross points between the line given by the resonance condition, $1-(16 / 5)\left(x^{\prime}-y \Delta u_{\mathrm{O}^{+5}} / V_{\mathrm{A}}\right)=0$, with the dispersion curve of a cold plasma with protons and electrons, which is $y^{2}=x^{\prime 2} /\left(1-x^{\prime}\right)$. In the wave number interval between the cross points we use simply the resonance condition instead of the dispersion relation (1), and on both side of the cross points we use the cold (proton and electron) plasma dispersion relation. The top left box in Fig. 1 shows a comparison between the exact solution of Eq. (1) (indicated by the thick dashed line) and the approximate solution (given by the continuous line). One can hardly tell the difference between the two solutions. Only in a very narrow range near to the cross point there is a little, but for our present purpose negligible, difference.

\section{Numerical results}

We first present the numerical result with a large spectrum density. At the boundary, the ratio between the wave power density at the oxygen resonance frequency and the background magnetic energy density is $f_{\mathrm{O}+5} P\left(f_{\mathrm{O}+5}\right) / B^{2}=210^{-4}$, where $P(f)$ is the magnetic spectrum power density and $f_{\mathrm{O}^{+5}}=\Omega_{\mathrm{O}^{+5}} / 2 \pi$. The corresponding magnetic power density near $160 \mathrm{~Hz}$ at $2.5 R_{\odot}$ is about $1000 \mathrm{nT}^{2} \mathrm{~Hz}^{-1}$. We notice that the power density presented by $\mathrm{Hu}$ et al. (1999) at the same frequency and 

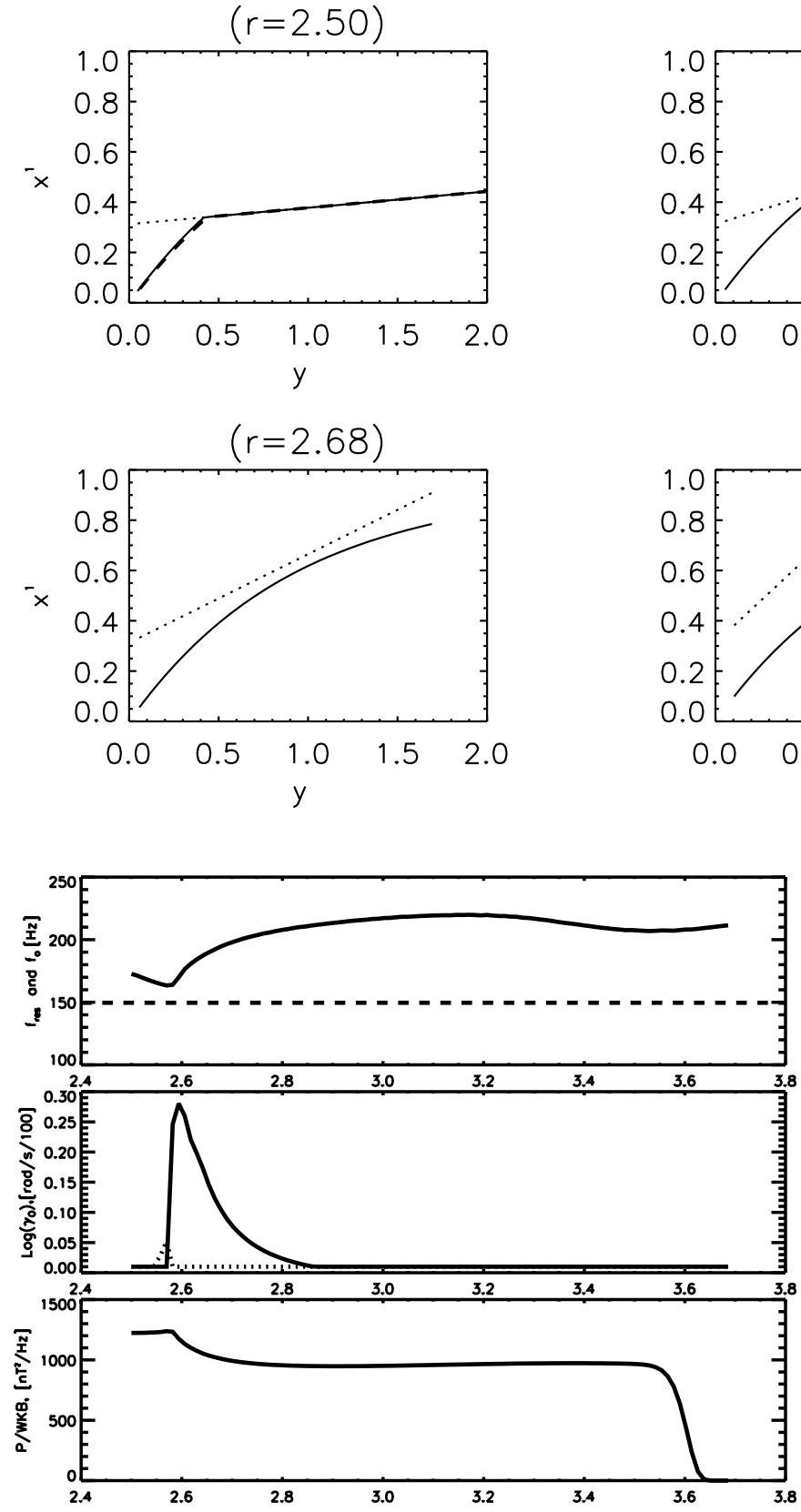

Fig. 2. Radial evolution of the resonant frequency $f_{\text {res }}$ (solid line in the top panel), the wave frequency $f_{0}$ in the inertial frame of reference (dashed line in the top panel), the damping rate caused by $\mathrm{O}^{+5}$ ions (middle panel, negative values shown by a solid line, positive values by a dotted line), and the normalized magnetic power density at $f_{0}$ (solid line in the bottom panel)

the same heliocentric distance is about $2000 \mathrm{nT}^{2} \mathrm{~Hz}^{-1}$ (see their Fig. 3).

Figure 1 shows the evolution of the dispersion relation and the resonance condition. Because the $\mathrm{O}^{+5}$ ions are quickly accelerated, their resonance line becomes steeper and finally (third frame for $r=2.68 R_{\odot}$ ) no longer crosses the dispersion curve any more. Because of the thermal broadening of the velocity distribution function, there is still a considerable number of $\mathrm{O}^{+5}$ ions in resonance with the cyclotron waves. Yet with increasing heliocentric distance, the resonance line becomes steeper and lies even further from the cold plasma dispersion curve. The thermal particles detach from cyclotron resonance, and as a result the dispersion curve attains the shape of that for a cold proton-electron plasma.

The radial evolution shown in Fig. 1 results in a decreasing damping rate of the waves. Figure 2 shows the associated evolution of the damping rate and the spectrum amplitude for a frequency $\left(f_{0}=150 \mathrm{~Hz}\right)$ at the boundary in the inertial frame of reference. In the top panel the solid line shows the radial variation of the "resonance frequency", $f_{\text {res }}=\left(\Omega_{\mathrm{O}^{+5}}+k\left(f_{0}\right) u_{\mathrm{O}^{+5}}\right) / 2 \pi$, which is the ion gyrofrequency Doppler shifted to the inertial frame. Here $k\left(f_{0}\right)$ is the wave number corresponding to the frequency $f_{0}$. The resonance condition may then be rewritten as $f_{\text {res }}=f_{0}$. The dashed line shows the radial profile of $f_{0}$, which is assumed to be constant. We see that with increasing heliocentric distance, near the boundary at $r=2.5 R_{\odot}, f_{\text {res }}$ decreases slightly. This is due to the decrease of $\Omega_{\mathrm{O}^{+5}}$ with decreasing magnetic field. After attaining its minimum value near $2.6 R_{\odot}, f_{\text {res }}$ again increases quickly, because of the increase of $u_{\mathrm{O}^{+5}}$ resulting from the fast acceleration. The wave with frequency $f_{0}$ is never resonant with the majority of the $\mathrm{O}^{+5}$ ions, because no cross point exists between the two lines.

However, near the minimum of $f_{\text {res }}$, a considerable fraction of $\mathrm{O}^{+5}$ ions is resonant with and thus causes damping of the waves. In the middle panel we show the associated damping rate owing to the resonance of the waves with $\mathrm{O}^{+5}$ ions (see the solid line). We see that the damping is concentrated in a narrow heliocentric distance range of size $0.1 R_{\odot}$. The bottom panel shows the radial variation of the power density normalized to the WKB factor at 


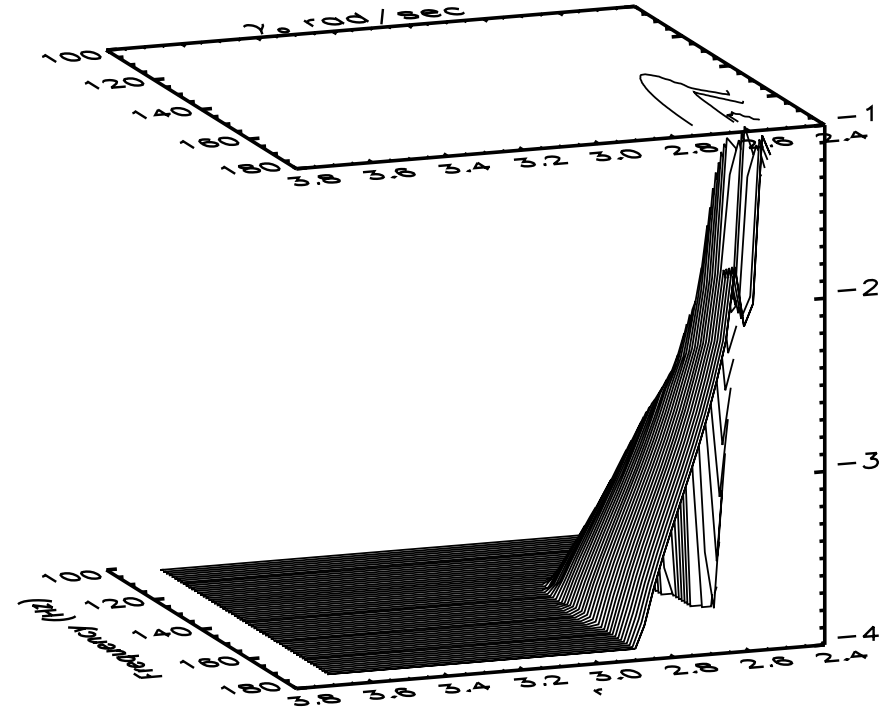

Fig. 3. The radial evolution of damping rate of the wave spectrum in the range from $100 \mathrm{~Hz}$ to $180 \mathrm{~Hz}$. This is a 3-D plot, where the $z$-axis shows $\log _{10}\left(-\gamma_{\mathrm{O}+5}[\mathrm{rad} / \mathrm{s}]+10^{-4}\right)$. The lines projected onto the top plane are the iso-contours of the damping rate

frequency $f_{0}$. Without resonance damping the power density would be constant. The WKB factor is unity at the boundary. The power density decreases by about 20 percent near $2.6 R_{\odot}$, then remains unchanged before being fully absorbed by the protons near $3.6 R_{\odot}$.

Figure 3 shows the damping rate caused by the $\mathrm{O}^{+5}$ ion resonance in a 3 -D plot with the $x$-axis giving the radial distance from 2.4 to $3.8 R_{\odot}$ and the $y$-axis showing the frequency from $100 \mathrm{~Hz}$ to $180 \mathrm{~Hz}$. The damping rate is plotted as a relief along the logarithmic $z$-axis. We see that the high damping rates, of about $0.1 \mathrm{rad} / \mathrm{s}$, are concentrated in a very small region of the plot near the lower-height and high-frequency boundary. Outside of this region, there is effectively no damping.

Figure 4 shows the evolution of the normalized spectrum under the influence of the damping at the rate shown in the previous Fig. 3. The power spectrum at $2.5 R_{\odot}$ is used as the input spectrum for the model calculation. The radial variation of the spectrum is obtained from the numerical analysis. For the heliocentric distance range from $2.5 R_{\odot}$ to $3.7 R_{\odot}$, the normalized spectrum density is almost constant in most parts of the plot. It only decreases a little near $r=2.6 R_{\odot}$ and then remains unchanged until being absorbed by protons near $3.6 R_{\odot}$. A slight increase of the power density appears at heights lower than those where the damping occurs. This power increase is caused by an instability driven by the thermal anisotropy that was assumed to exist at the lower boundary.

The wave damping becomes severe if less initial wave power density is assumed at the lower boundary. Figure 5 shows results for $f_{\mathrm{O}^{+5}} P\left(f_{\mathrm{O}^{+5}}\right) / B^{2}=210^{-5}$. This value was also used in Model 3 of Tu \& Marsch (2001). The power density at $r=2.5 R_{\odot}$ and at $160 \mathrm{~Hz}$ is about $100 \mathrm{nT}^{2} \mathrm{~Hz}^{-1}$. We see that the damping of the waves

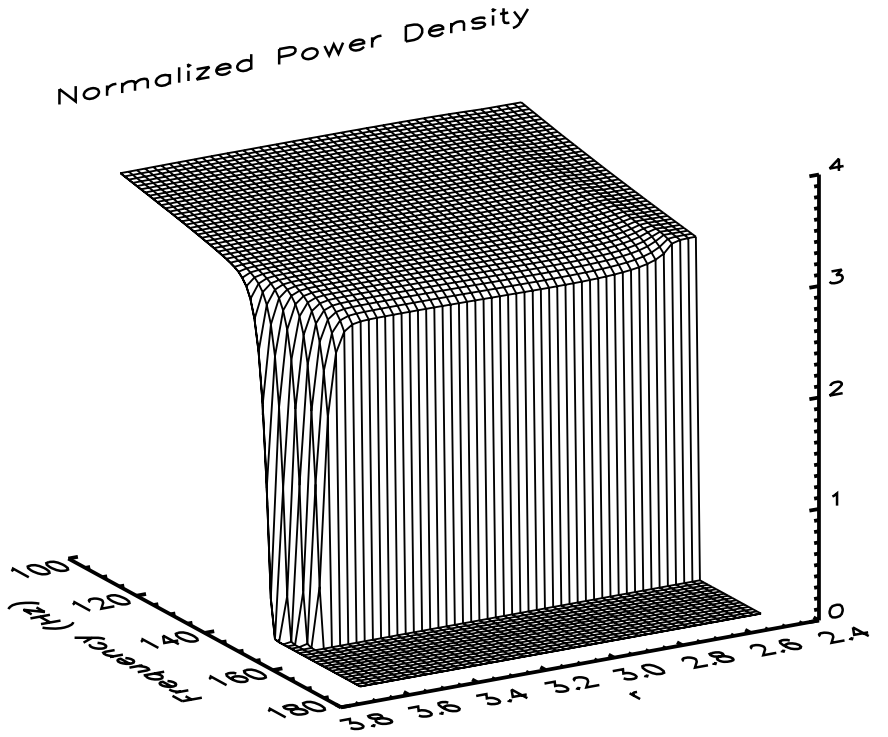

Fig. 4. Radial evolution of the power spectrum normalized to the WKB value. The $z$-axis of the 3 -D plot shows $\log _{10}\left(P(r, f) /\left[\mathrm{nT}^{2} \mathrm{~Hz}^{-1} \mathrm{WKB}\right]+1\right)$. The $x$-axis shows the radial distance range from $2.5 R_{\odot}$ to $3.7 R_{\odot}$, and the $y$-axis shows the frequencies from $100 \mathrm{~Hz}$ to $180 \mathrm{~Hz}$

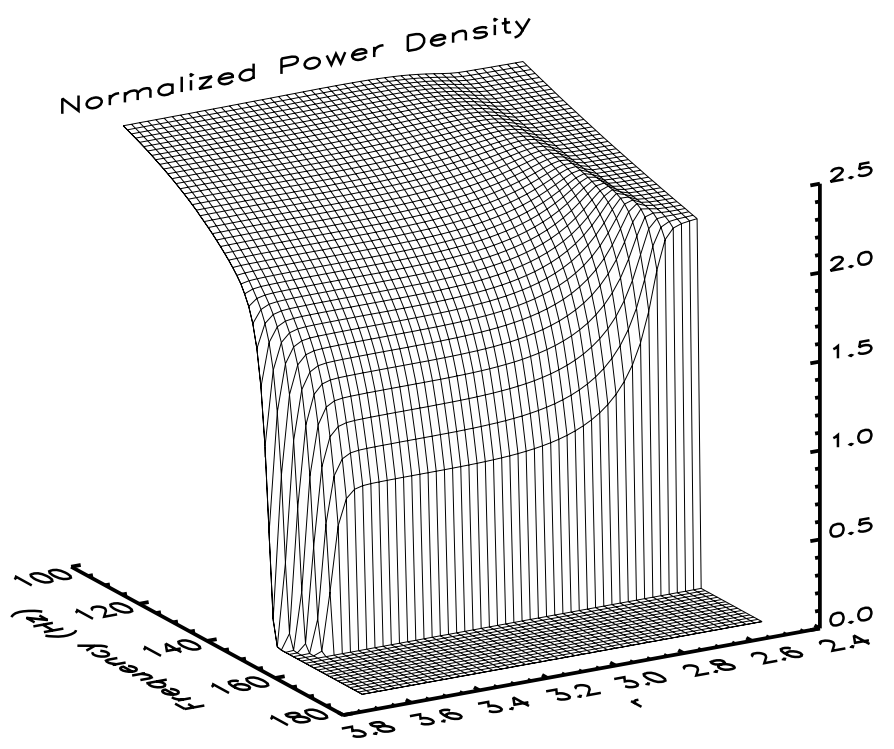

Fig. 5. Same format as in Fig. 4, but here with a spectrum density being by one order of magnitude lower at $r=2.5 R_{\odot}$, i.e. being about $100 \mathrm{nT}^{2} \mathrm{~Hz}^{-1}$

is more pronounced and extends over a wider frequency domain.

Finally, Fig. 6 shows a case with extremely low relative wave power density of only $f_{\mathrm{O}^{+5}} P\left(f_{\mathrm{O}^{+5}}\right) / B^{2}=210^{-6}$. The power density at $r=2.5 R_{\odot}$ and at $160 \mathrm{~Hz}$ is about $10 \mathrm{nT}^{2} \mathrm{~Hz}^{-1}$. We see a severe erosion of wave power appearing at frequencies from $120 \mathrm{~Hz}$ to $160 \mathrm{~Hz}$. If one would allow for wave absorption by many heavy ions from many chemical elements simultaneously (see Cranmer 2000), probably not much wave energy would be left over for the majority of the solar wind protons to absorb. 


$$
\text { Normalized Power Density }
$$

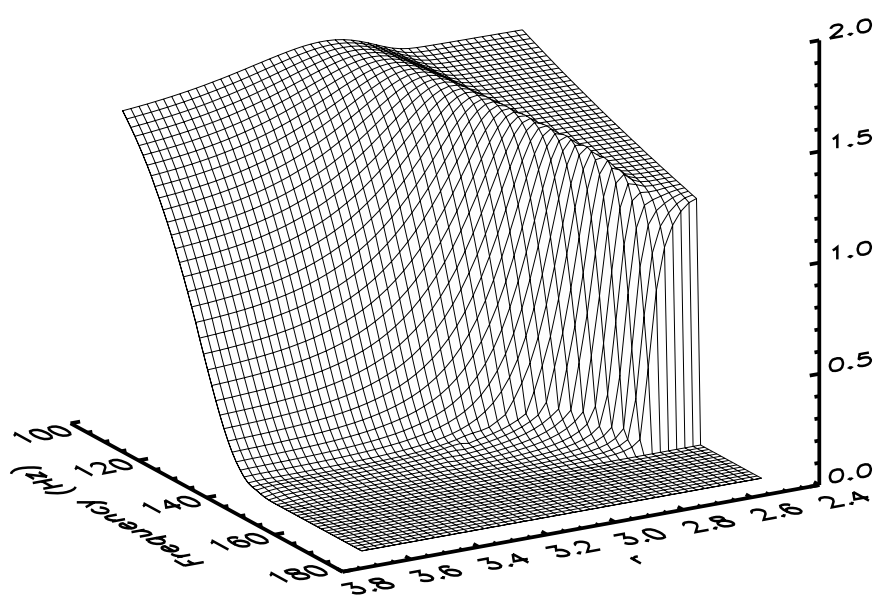

Fig. 6. Same format as in Fig. 4, but here with a spectrum density two orders of magnitude lower at $r=2.5 R_{\odot}$, i.e. about $10 \mathrm{nT}^{2} \mathrm{~Hz}^{-1}$

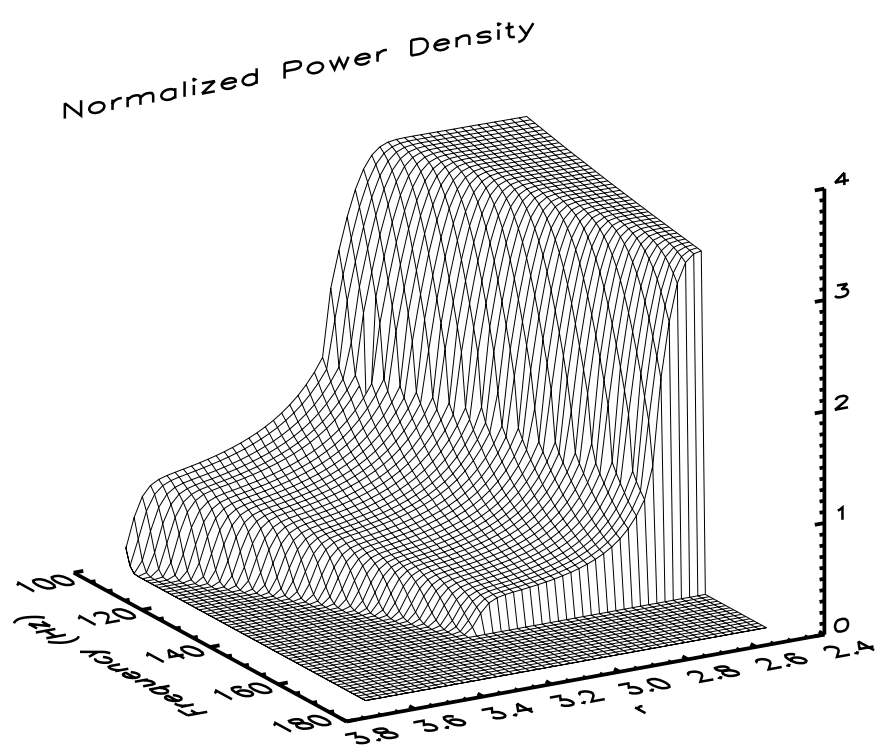

Fig. 7. Same format as in Fig. 4, but here with fixed velocities: $u_{\mathrm{O}^{+5}}=340 \mathrm{~km} \mathrm{~s}^{-1}, v_{\|, \mathrm{O}^{+5}}=260 \mathrm{~km} \mathrm{~s}^{-1}$, and $v_{\perp, \mathrm{O}^{+5}}=$ $120 \mathrm{~km} \mathrm{~s}^{-1}$

Figure 7 shows the numerical result with the same wave power density at the boundary as was used in Fig. 4 , but here we assume that all velocities, $u_{\mathrm{O}^{+5}}=340 \mathrm{~km} \mathrm{~s}^{-1}$, $v_{\|, \mathrm{O}^{+5}}=260 \mathrm{~km} \mathrm{~s}^{-1}$, and $v_{\perp, \mathrm{O}^{+5}}=120 \mathrm{~km} \mathrm{~s}^{-1}$, remain unchanged with heliocentric distance. We see that the wave power density suffers severe damping at all frequencies. Less than one percent of the energy is left for protons to absorb. Apparently, the tiny number of $\mathrm{O}^{+5}$ ions can absorb an infinite amount of energy, because the constant velocity assumption is not self-consistent and thus enables the particles to continuously absorb wave energy.

\section{Discussion and conclusions}

The numerical results of the model, with a self-consistent treatment of ion acceleration and wave damping, show that the portion of the wave energy absorbed by the ions during their cyclotron resonance depends strongly on the value of the power density put in at the lowerheight boundary. If the wave power density is large, say $1000 \mathrm{nT}^{2} \mathrm{~Hz}^{-1}$, the wave damping is small and concentrated in a very narrow frequency band, which is about $10 \mathrm{~Hz}$ wide. Even when one considers many ions from different minor species, the major part of the wave energy would be left for the solar wind ions (protons and alpha particles) to absorb. However, if the wave power density is extremely low, say only $10 \mathrm{nT}^{2} \mathrm{~Hz}^{-1}$, then the damping by minor ions is severe and the spectrum is almost fully eroded. Not much energy is left in this case for solar wind majority species to absorb.

Our model calculation was designed to clarify this important issue, but was not intended to give a full description of the couplings and interactions between ions and waves. Our model calculation with a large wave power density produced too high an $\mathrm{O}^{+5}$ velocity, with $u_{\mathrm{O}^{+5}}=1400 \mathrm{~km} \mathrm{~s}^{-1}$. This may be related to using the cold plasma dispersion relation in that model, following Cranmer (2000). When we used the thermal-plasma dispersion of Model 3 of Tu \& Marsch (2001), we obtained reasonable heating of both the $\mathrm{O}^{+5}$ ion and proton species, in accord with observational values. Our investigation clearly showed that one has to carefully take bulk drifts as well as temperature anisotropies into account if one wants to evaluate cyclotron-wave absorption. By drifting, the bulk of the heavy minor ions can detune effectively from the resonance, whereby the plasma in its minor ion component becomes transparent for the waves. They can thus be absorbed by the majority ions (the protons). Plasma wave transfer and opacity thus depend in a subtle way on the ion velocity distribution function. To develop a satisfying model fully describing the resonant interactions between the different ion species and the cyclotron waves still needs a lot of effort. This will require consideration of the complete quasilinear theory and sophisticated kinetic numerical calculations to be carried out.

Acknowledgements. Parts of Tu's work were supported by the National Natural Science Foundation of China under projects with contract numbers 49874036 and 49990452.

\section{References}

Axford, W. I., \& McKenzie, J. M. 1997, in Cosmic Winds and the Helisophere, ed. M. S. Matthews, A. S. Ruskin, \& M. L. Guerrieri (The University of Arizona Press, Tucson, AZ, USA), 31

Cranmer, S. R. 2000, ApJ, 532, 1197.

Cranmer, S. R., et al. 1999, ApJ, 511, 481.

Gomberoff, L., Gratton, F. T., \& Gnavi, G. 1996, J. Geophys. Res., 101, 15661 
Hackenberg, P., Mann, G., \& Marsch, E. 1999, Space Sci. Rev., 87, 207

Hackenberg, P., Marsch, E., \& Mann, G. 2000, A\&A, 360, 1139

Hu, Y.-Q., Habbal, S. R., \& Li, X. 1999, J. Geophys. Res., 104, 17045

Kohl, J. L., Noci, G., Antonucci, E., et al. 1998, ApJL, 501, L127

McKenzie, J. F., Banaszkiewicz, M., \& Axford, W. I. 1995, A\&A, 303, L45

McKenzie, J. F., Axford, W. I., \& Banaszkiewicz, M. 1997, Geophys. Res. Lett., 24, 2877

Marsch, E., \& Tu, C.-Y. 1997a, A\&A, 319, L17

Marsch, E., \& Tu, C.-Y. 1997b, Solar Phys., 176, 87
Marsch, E., Goertz, C. K., \& Richter, K. 1982, J. Geophys. Res., 87, 5030

Spangler, S., \& Manusco, S. 2000, ApJ, 530, 491

Tu, C.-Y., \& Marsch, E. 1997, Solar Phys., 171, 363

Tu, C.-Y., \& Marsch, E. 1999, in Solar Wind Nine, ed. S. R. Habbal, et al., AIP Conf. Proc., 471, 373

Tu, C.-Y., \& Marsch, E. 2001, J. Geophys. Res., in press

Tu, C.-Y., Marsch, E., Wilhelm, K., et al. 1998, ApJ, 503, 475

Tu, C.-Y., Marsch, E., \& Wilhelm, K. 1999, Space Sci. Rev., 87, 331

Wilhelm, K., Marsch, E., Dwivedi, B. N., et al. 1998, ApJ, 503, 1023 